\title{
Mechanistic mechanisms of competition and biodiversity
}

\author{
Lev V. Kalmykov ${ }^{1,2}$, Vyacheslav L. Kalmykov ${ }^{2,3}$ \\ ${ }^{1}$ Institute of Theoretical and Experimental Biophysics, Russian Academy of Sciences, Pushchino, Moscow Region, \\ 142290 Russia. \\ 2Pushchino State University, Pushchino, 142290, Moscow Region, Russia. \\ ${ }^{3}$ Institute of Cell Biophysics of the Russian Academy of Sciences, Pushchino, 142290, Moscow Region
}

\section{Abstract}

The nature of competition and biodiversity are open basic questions since Darwin. To investigate mechanisms of interspecific competition and their contribution in biodiversity as closely as possible we offer a white-box modelling method based on physically interpreted ecological axioms. These models are implemented as deterministic individual-based cellular automata and able to give a direct physicomechanistic insight into studied phenomena. Competition of two trophically identical but fitness different species, competing for one limiting resource in one stable uniform habitat (which is closed for immigration, emigration, predation, herbivory and parasitism) has been investigated in conditions, which are the most unfavourable for their coexistence. The species are per capita identical in fecundity, ontogeny, regeneration features of microhabitats, and in habitat requirements. We have modelled following 8 mechanistic mechanisms of interspecific competition:

1. A case of the competitive exclusion when competing species differ only in fitness.

2. Coexistence based on periodic dominance changeovers as a consequence of environmental changes. Competing species differ only in fitness.

3. A strong violation of the competitive exclusion principle due to the lowered fecundity of both competitors. Competing species differ only in fitness.

4. Coexistence based on the competition-colonisation trade-off when greater fitness is compensated by r-strategy.

5. A competition-colonisation trade-off based on differences in ontogeny.

6. Competitive exclusion when recessive species drives out the dominant one having four times greater fecundity than the dominant one in stable environment (the greater fitness cannot compensate r-strategy).

7. An inverted competitive exclusion when recessive species drives out the dominant one by strategy of anticipatory deprivation of resources for competitor's offsprings propagation. Recessive species drives out the dominant one in stable environment and both competing species have identical fecundity (tripod neighbourhood). Paradoxically, but the greater fitness cannot save the dominant species when the all other parameters of the species are equal.

8. Both competing species die because the regeneration of a limiting environmental resource takes too much time and they cannot propagate. 
The revealed mechanisms of competition can be useful not only in conservation biology, but also in economics and politics. Additionally, we speculate that the simplest way to maintain biodiversity is a controlled reduction of human fertility as the decrease in biodiversity occurs largely due to humankind overloading of biosphere resources.

Keywords: white-box model, mechanistic mechanism, biodiversity, competition, conservation biology, cellular automata, duopoly.

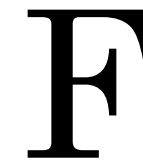

ield observations of interspecific competition, laboratory researches and theoretical interpretations are often poorly join together, because they correspond to different time scales, to various experimental conditions and to different scientific approaches (1). Arising misinterpretations have contributed to the biodiversity paradox. The Gauze's competitive exclusion principle $(1,2)$ belongs to the one side of this paradox, while the paradox of the plankton (3) together with at least 120 different hypotheses (4) of natural species richness are on the other side (5-8). In the Gauze's experiments many generations of the competing species replaced one another in the stable laboratory environment (2). The facts of the paradox of the plankton were received in complex plankton communities of the natural reservoirs with changeable environmental conditions. The rainforests data, underlying the neutral theory (7), concern to a short period of life of tropical trees and are associated with a very open ecosystem with unknown environmental stability on previous long periods.

Tilman drew attention to the prevalence of nonmechanistic Lotka-Volterrabased understanding of competition - most ecologists have studied competition by asking if an increase in the density of one species leads to a decrease in the density of another, without asking how this might occur (9). Physical mechanisms of biodiversity suppose possibility of use of extreme principles of physics. Earlier Lotka postulated that in result of natural selection the entire system of living organisms evolves under a stream of environmental energy in direction of increase of: (i) bioproductivity, (ii) metabolism rate and (iii) the total energy flux through the system (10). The 'energy flux' is the environmental energy, which is absorbed and dissipated within the ecosystem per unit time during their life activity. As environmental conditions are constantly changing, the greater will be biodiversity of an ecosystem, the greater will be bioproductivity, metabolism rate and energy flux. A species that currently has a lower fitness and is recessive may become dominant due to change of environmental conditions (Fig. 1). Models of competition predict, and field experiments confirm, that greater plant diversity leads to greater primary productivity (11). The generalized mathematical formalism of evolutionary generation of biodiversity was proposed earlier by one of the authors (12). According to this formalism, evolution is the way of realization of extreme principles of physics 
through permutation of integral co-organization of elements of an ecosystem. A choice of trajectory of most effective using of environmental energy occurs spontaneously in according to principle of a dissipative self-organization (13). Tansley was the first who coined the term ecosystem as the fundamental unit of ecological organization in the sense of physics (14). R. Lindeman (15) and Eugene P. Odum (16) popularized and further developed the framework of ecosystem ecology. The important conclusion was that the ecosystem as a whole is object of applicability of extreme principles of physics $(10,12,14)$. The second law of thermodynamics is the main extreme principle of physics. In ecology this principle manifests itself through interspecific competition for environmental resources (17, 18).

Here the interspecific competitive fitness is defined through dominance. The dominance we define as the primary ability of individuals of the species to occupy a free microhabitat in a conflict of interests with an individual of another species of a species in of a concrete microhabitat resource using. It should be also noted that population autowaves' patterns(19) are dissipative structures (20) whose dynamics is clearly related to the implementation of the extreme principles. Here we offer the deterministic, individual-based cellular-automata method to reveal mechanistic mechanisms of competition. The method provides reducing of complex mechanisms of holistic ecosystem's dynamics to the level of simple logical axioms.

Earlier many separate explanations of biodiversity paradox have been supposed, however generalized mechanisms were not offered $(5,21,22)$. Our method allows revealing and unequivocally to interpret mechanisms of interspecific competition because it is based on models, which are the logic-inference automata of deterministic cause-and-effect logic conclusions. Detailed spatio-temporal dynamics of each individual as and interconnected dynamics of microhabitats, minihabitats, and a whole macrohabitat are reproduced in details. The cellular automata allow to create simple models of complex nonlinear dynamic systems by the most simple and natural way.

The first investigated model of competition reproduces situation when environment is stable and individuals of both species aggressively propagate in all adjacent cells. Individuals of both species propagate in hexagonal neighborhoods on hexagonal lattice. In this model, the dominant species completely drives out the recessive one (Fig. $1 \mathrm{~A}$ and movie $\mathrm{S} 1$ ). We define dominance as the primary ability of an individual of a dominant species to occupy a free microhabitat in conflict of interests with an individual of a recessive species in result of greater ecological fitness (fig. S4, A and B). The fact that under stable conditions of external and internal environment only dominant species survives, confirms the principle of competitive exclusion. The results in Fig. 1A conform to the experimental data of Gauze (2) relating to competition in the mixed populations of Paramecium 
caudatum and Paramecium aurelia in the medium with the lowest concentration of food resources.
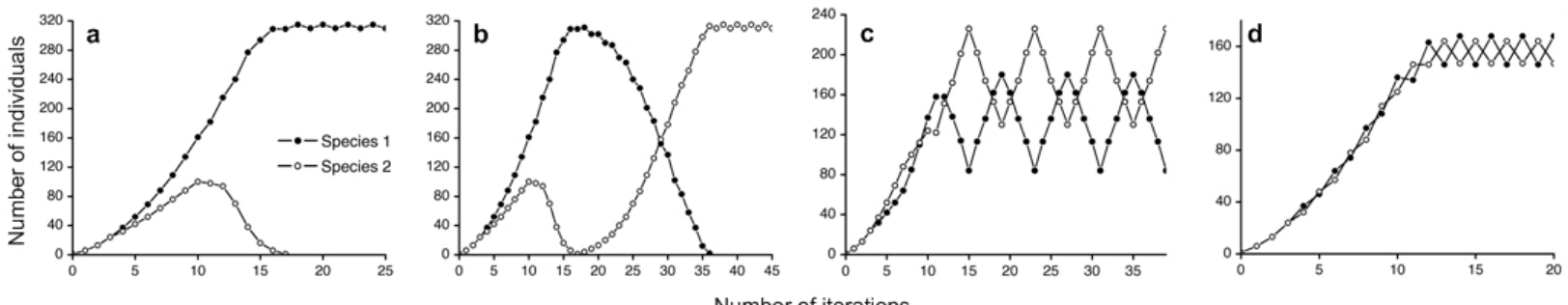

Fig. 1. Population dynamics. Individuals of the both competing species have the same fecundity (hexagonal neighborhood). (A) Stable environmental conditions. The species ' 1 ' is dominant. See movie S1. (B) The single changeover of dominance after environmental change at the eighteenth iteration. (C) Periodic changeovers of dominance after environmental changes at each fourth iteration of the cellular automaton. (D) Periodic changeovers of dominance after environmental changes at each iteration of the cellular automaton. See movie S2.

Due to differences in adaptation to various environmental factors, a recessive species can becomes dominant after environmental change. Such exchange of a competitiveness status we called 'changeover of dominance'. Three models with this mechanism are presented in Fig. 1, B to D. The single changeover of dominance on the eighteenth iteration of the cellular automaton leads to the prolongation of coexistence and to subsequent extinction of the initially dominant species (Fig. 1B). Periodic changes of environmental conditions on each fourth iteration, which are accompanied by changeovers of dominance, provide a coexistence of the competitors (Fig. 1C). Periodic changeovers of dominance on each iteration of the cellular automaton maintain the most stable coexistence (Fig. 1D). With more frequent repetitions of changeover of dominance the sustainability of coexistence is better. The possibility of existence of the similar mechanism of biodiversity was indicated by Hutchinson in 1941 (23). He suggested that the reasons of diversity of phytoplankton are environmental fluctuations (e.g. fluctuations of weather) which block the implementation of the competitive exclusion principle. 

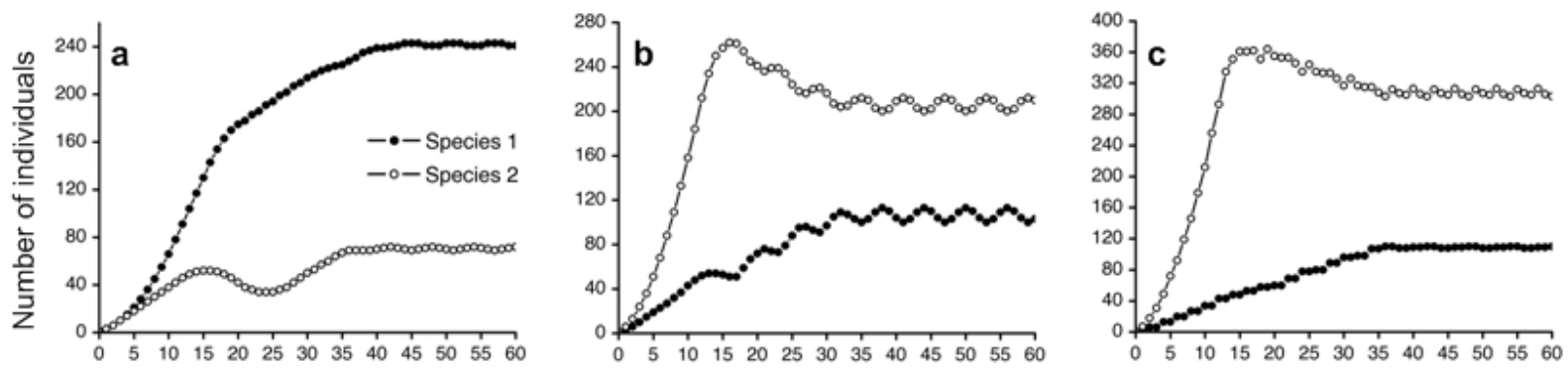

Number of iterations

Fig. 2. A competitive coexistence in stable environment. The species ' 1 ' is dominant. (A) A strong violation of the Gauze's principle of competitive exclusion due to the low competitors' fecundity. Individuals of the competing species propagate in the tripod neighborhood on the hexagonal lattice (movie S3). (B) A competition-colonization trade-off. Individuals of the competing species have different fecundities. Individuals of the species ' 1 ' propagate in the tripod neighborhood. Individuals of the species ' 2 ' propagate in the hexagonal neighborhood. (C) A competition-colonization trade-off, based on differences of ontogenetic stages. Both species have hexagonal neighborhoods and two ontogenetic stages. The dominant species propagates only in the second ontogenetic stage, and the recessive species propagates in the both stages.

In this investigation we look for the answer to the same question which was investigated by Gauze (2). The question is: Is it possible the coexistence of two similar species, for verification of the principle we formulated so: Is it possible a stable coexistence of competing for the same resource in one uniform habitat with limited resource, when 'one species has any advantage over the other' and when environmental conditions are stable? We try to eliminate possible pitfalls of this issue. Therefore, the focus of this paper concentrated on formulation of the extremely rigorous conditions for testing the principle of competitive exclusion in the context of various mechanisms of competition. This strong question for verification of the principle we formulated so: Is it possible a stable coexistence of two similar (but different) the genetically stable-homogeneous species, competing for one limiting resource in one stable uniform habitat (which is closed for immigration, emigration, predation, herbivory and parasitism), if the species are per capita identical in fecundity, ontogeny, regeneration features of microhabitats, and in habitat requirements (are trophically identical consumers), but different in fitness (one species is dominant in a direct competition for a microhabitat resource)? The positive answer to this question we named the strong violation of the Gauze's principle. The all methodological features of this work have been developed to ensure the correctness of verification of the principle. 
The competition dependence on fecundity features of species is presented in Fig. 2. Fecundity rates are simulated by varying the types of propagation neighborhoods. Possibilities of various neighborhoods using for modeling of various fecundity rates are demonstrated in fig. S2. Fecundity decreasing is modeled by decreasing the number of cells in the neighborhood. For this purpose, we used tripod neighborhood on the hexagonal lattice for the both competitors. Tripod neighborhood is equal to half of the standard hexagonal neighborhood (figs S1 and S6). The propagation in tripod neighborhood simulates what an individual may use for propagation only a half of resources of nearest surrounding. The results of this experiment demonstrate the strong violation of the Gauze's principle of competitive exclusion.

The case of coexistence that is presented in Fig. 2A and movie S3 is possible because (i) offsprings of each individual of both species occupy only half of the nearest surrounding resources and (ii) due to the tunnel penetration of offsprings of the recessive species through gaps in population autowaves of the dominant species (figs S1 and S3). The results of this model demonstrate the strong violation of the Gauze's principle. It seems reasonable to say that this strict deterministic theoretical conclusion is of greater importance for TO falsification of the Gauze's principle, than any practical experiment, because of the point of view that any empirical studies cannot prove and hardly falsify the Gauze's principle (1).

The next two models show the competitive coexistence of two species as result of competition-colonization trade-offs - the species differ in fitness (one of them is dominant) and fecundity (Fig. 2, B and C). The first of these two models (Fig. 2B) shows that the double increasing of fecundity rate of the recessive species provides competitive coexistence of species. In the second model, the coexistence is based on differences of competitors in ontogenetic features of fecundity and in fitness (Fig. $2 \mathrm{C}$ ). The both species have one additional ontogenetic life stage. The dominant species propagates only on the second ontogenetic stage and recessive species propagates on the both stages. The detailed results of competition of two species with various fecundities (based on differences in neighbourhoods and on different features of ontogenetic stages) are presented in table $\mathrm{S} 2$. The concept of ontogenetic stages in cellular automata models of plant populations was proposed earlier by Komarov (24). 


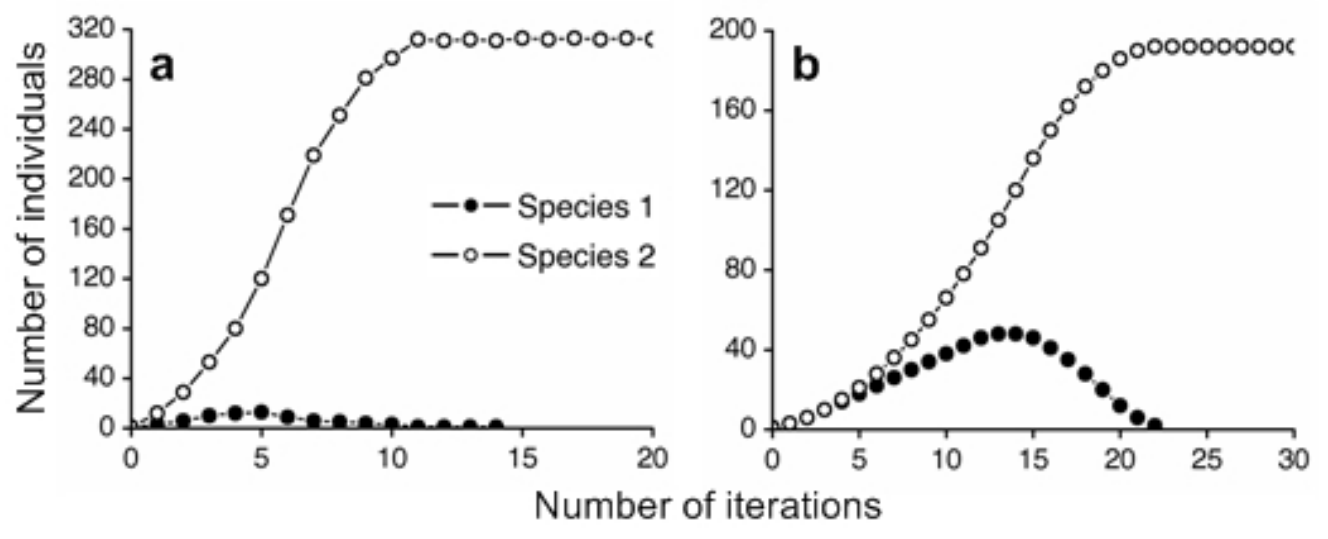

Fig. 3. Recessive species drives out the dominant one in stable environment. The species ' 1 ' is dominant. (A) The recessive species has four times greater fecundity than the dominant one. The dominant species propagates into the tripod neighborhood (three offsprings) and the recessive species propagates into the neighborhood with twelve cells. (B) Both competing species have identical fecundity (tripod neighborhood). Nonstandard parameters: the lattice size is of $24 \times 24$ cells and coordinates of the initiating individual of the recessive species are $(14,13)$. See movie S4.

The results represented in Fig. 3A show the case of competitive exclusion, which is based on the fourfold fecundity rate of the recessive species. The recessive species in consequence of considerably greater fecundity wins the competition with the dominant one. It is the situation of competition when the r-strategy beats the Kstrategy (25). The recessive species also may win competition with the dominant species and with the same fecundity due to strategically advantageous positioning (Fig. 3B and movie S4). Owing to strategically advantageous positioning, individuals of the recessive species propagate into microhabitats just before the moment when individuals of the dominant species will try to occupy these microhabitats on the next iteration. This strategy allows individuals of the recessive species avoid direct competition and to predeprive the dominant species of resources for propagation. Such a strategy is evolutionarily the most promising because it allows competing successfully to with a stronger competitor having the same fecundity. Implementing of this strategy depends not only on the basic rules, but also on the lattice size and the initial placement of individuals. The recessive species wins due to gaps in population autowaves of the dominant species. The gaps arise owing to double reduction of fecundity rates (propagation in tripod neighborhood on the hexagonal lattice - fig. S1B). On the 22th iteration of the possibility of propagation of the last individuals of the dominant species are completely blocked, and on the 23th iteration the dominant species is eliminated 
(Fig. 3B and movie S4). The gaps in segmented population autowaves (fig. S1B) underlie the mechanisms of the models presented in Fig 2, A and B, and Fig. 3, including strong violation of Gauze's principle and strategically advantageous positioning of offsprings. These gaps arise due to lowering of fecundity rate of a species. The hexagonal neighborhood provides closed fronts of population autowaves (fig. S1A). The closed population autowaves of the dominant species does not give possibility for individuals of recessive species to get through the autowaves fronts of the dominant species. The tripod neighborhood on the same hexagonal lattice simulates a lower fecundity rate and forms the gap-segmented fronts of population autowaves (fig. S1, S2 and S7). Half of resources of nearest environment remains untouched in case of the wave front with gaps. These free resources are the 'gates' for penetration of offsprings of the recessive species. Individuals of the recessive species pass through fronts of autowaves population waves of the dominant species and further through the periodic boundary conditions the competing species equilibrate each other. One example of such coexistence of two ecologically similar competing species (the strong violation of the Gauze's principle) is shown in fig. S3. It is the pattern of the tunnel propagation of individuals of the recessive species through the gaps in population autowaves of the dominant one. The applicability of our method to different disciplines is demonstrated on examples of the lawn grasses competition, an economic duopoly (Supplementary Information text).

The decrease in biodiversity occurs largely due to human activities. Overloading of biosphere resources disturbs their natural regeneration. With the appearance of man, which is the universal consumer, the same extreme principles of physics have become a source of biodiversity loss. The human overpopulation replaces populations of wildlife species. Natural selection in nature is replaced by human creativity and by industrial exploitation of natural resources. Human activity leads to greater energy flux through biosphere, in comparison with energy flux generated by wildlife, i.e. the humanity dissipates energy more actively. It is in interest of humanity to save the largest possible number of existing species for ensuring the sustainability of ecosystems and for providing of needs of the future biotechnology. Conservation of biodiversity can be achieved as by creating more environmentally friendly technologies, so and by reducing of biosphere resources overloading. The simplest way to maintain biodiversity is a controlled reduction of human fertility as the decrease in biodiversity occurs largely due to humankind overloading of biosphere resources $(26,27)$. 


\section{References}

1. G. Hardin, The competitive exclusion principle. Science 131, 1292 (Apr 29, 1960).

2. G. F. Gause, The struggle for existence. (NY: Hafner Publishing Company, New York, 1934), pp. ix, 163 p. incl. illus., tables, diagrs.

3. G. E. Hutchinson, The paradox of the plankton. Am. Nat. 95, 137 (1961).

4. M. W. Palmer, Variation in Species Richness - Towards a Unification of Hypotheses. Folia Geobot Phytotx 29, 511 (1994).

5. J. B. Wilson, Mechanisms of Species Coexistence - 12 Explanations for Hutchinson Paradox of the Plankton - Evidence from New-Zealand PlantCommunities. New Zealand Journal of Ecology 13, 17 (1990).

6. U. Sommer, Ecology - Competition and coexistence. Nature 402, 366 (Nov 25, 1999).

7. S. P. Hubbell, The unified neutral theory of biodiversity and biogeography. Monographs in population biology ; 32 (Princeton University Press, Princeton, N.J. ; Oxford, 2001), pp. xiv, 375 p.

8. J. Whitfield, Neutrality versus the niche. Nature 417, 480 (May 30, 2002).

9. D. Tilman, The Importance of the Mechanisms of Interspecific Competition. The American naturalist 129, 769 (1987).

10. A. J. Lotka, Contribution to the Energetics of Evolution. Proceedings of the National Academy of Sciences of the United States of America 8, 147 (Jun, 1922).

11. D. Tilman, The ecological consequences of changes in biodiversity: A search for general principles. Ecology 80, 1455 (Jul, 1999).

12. V. L. Kalmykov, in Evolutionary Computing, D. Corne, J. Shapiro, Eds. (Springer Berlin / Heidelberg, 1997), vol. 1305 of Lecture Notes in Computer Science, pp. 43-51.

13. E. Jantsch, in Autopoiesis, a theory of living organizations, M. Zeleny, Ed. (North Holland, New York, N.Y., 1981), vol. 3 of North Holland Series in General Systems Research, pp. 65-88.

14. A. G. Tansley, The Use and Abuse of Vegetational Concepts and Terms. Ecology 16, 284 (1935).

15. R. L. Lindeman, The Trophic-Dynamic Aspect of Ecology. Ecology 23, 399 (1942).

16. E. P. Odum, The Strategy of Ecosystem Development. Science 164, 262 (April 18, 1969, 1969).

17. L. Boltzmann, Ed., The second law of thermodynamics, 1886; reprinted in Ludwig Boltzmann, Theoretical Physics and Philosophical Problems, B. McGuinness, Ed. (Reidel Pub. Co., Dordrecht; Boston, 1974), Vol. 5 of Vienna circle collection, (Dordrecht ; Boston, 1974), pp. 13-32.

18. G. F. Gauze, The struggle for existence. (The Williams \& Wilkins company, Baltimore, 1934), pp. ix, 163 p. incl. illus., tables, diagrs.

19. G. R. Ivanitsky, A. B. Medvinsky, M. A. Tsyganov, From Cell-Population Wave Dynamics to Neuroinformatics. Uspekhi Fizicheskikh Nauk 164, 1041 (Oct, 1994).

20. I. Prigogine, Time, Structure, and Fluctuations. (1978), vol. 201, pp. 777-785. 
21. J. S. Clark et al., Resolving the biodiversity paradox. Ecology Letters 10, 647 (Aug, 2007).

22. J. Silvertown, Plant coexistence and the niche. Trends in Ecology \& Evolution 19, 605 (Nov, 2004).

23. G. E. Hutchinson, Ecological aspects of succession in natural populations. Am. Nat. 75, 406 (1941).

24. A. S. Komarov, M. M. Palenova, O. V. Smirnova, The concept of discrete description of plant ontogenesis and cellular automata models of plant populations. Ecological Modelling 170, 427 (Dec 15, 2003).

25. E. R. Pianka, On $r$ - and $K$-selection. American Naturalist 104, 592 (1970).

26. P. M. Vitousek, H. A. Mooney, J. Lubchenco, J. M. Melillo, Human Domination of Earth's Ecosystems. Science 277, 494 (July 25, 1997, 1997).

27. R. P. Cincotta, J. Wisnewski, R. Engelman, Human population in the biodiversity hotspots. Nature 404, 990 (Apr 27, 2000).

28. M. D. Casler, R. R. Duncan, Turfgrass biology, genetics, and breeding. (John Wiley \& Sons, Inc., Hoboken, N.J., 2003), pp. x, 367 p.

29. T. Bell, R. P. Freckleton, O. T. Lewis, Plant pathogens drive density-dependent seedling mortality in a tropical tree. Ecol Lett 9, 569 (May, 2006).

30. M. Scheffer, S. Rinaldi, J. Huisman, F. J. Weissing, Why plankton communities have no equilibrium: solutions to the paradox. Hydrobiologia 491, 9 (Jan, 2003).

31. S. Roy, J. Chattopadhyay, Towards a resolution of 'the paradox of the plankton': A brief overview of the proposed mechanisms. Ecological Complexity 4, 26 (Mar, 2007).

32. D. Tilman, The importance of the mechanisms of interspecific competition. The American naturalist 129, 769 (1987).

33. S. E. Jørgensen, G. Bendoricchio, Fundamentals of ecological modelling. Developments in environmental modelling (Elsevier, Amsterdam; New York, ed. 3rd, 2001), pp. xii, 530 p.

34. A. Kroll, Grey-box models: Concepts and application. In: New Frontiers in Computational Intelligence and its Applications, vol.57 of Frontiers in artificial intelligence and applications. M. Mohammadian, Ed., (IOS Press, Amsterdam, 2000).

35. M. Jelali, A. Kroll, Hydraulic servo-systems : modelling, identification, and control. Advances in industrial control (Springer, London; New York, 2003), pp. $\mathrm{xxx}, 355 \mathrm{p}$.

36. B. Roffel, B. H. Betlem, Advanced practical process control. Advances in soft computing (Springer, Berlin ; New York, 2004), pp. ix, 309 p.

37. S. Estrada-Flores, I. Merts, B. De Ketelaere, J. Lammertyn, Development and validation of "grey-box" models for refrigeration applications: A review of key concepts. International Journal of Refrigeration 29, 931 (2006).

38. A. Engel, Verification, validation, and testing of engineered systems. Wiley series in systems engineering and management (Wiley, Hoboken, N.J., 2010), pp. xix, $687 \mathrm{p}$. 
Acknowledgements We thank A.S. Komarov for inspiration of our interest to the subject. We thank G.Yu. Riznichenko and L.G. Khanina for discussion and comments, O.V. Vykidanets and A.E. Malafeeva for some language corrections. We thank A.V. Kalmykov for discussion, comments and idea of the Glossary of terms.

Author Contributions V.L.K. designed the research. L.V.K. created the programs, investigated the models and made the figures and the movies of the experiments. Both authors discussed the results, interpreted them and wrote the manuscript.

Correspondence and requests for materials should be addressed to V.L.K. (vyacheslav.l.kalmykov [at] gmail.com). 


\section{SUPPLEMENTARY INFORMATION}

a
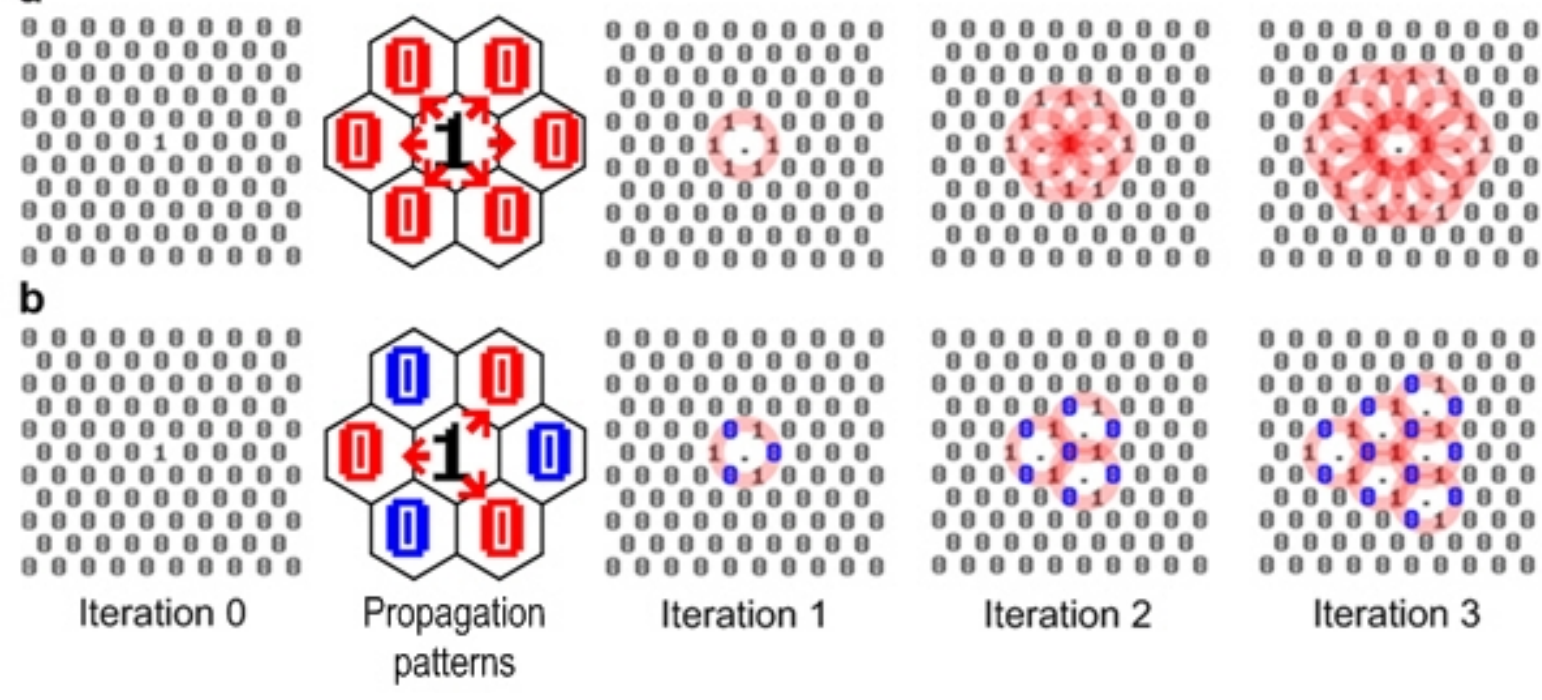

Iteration 2

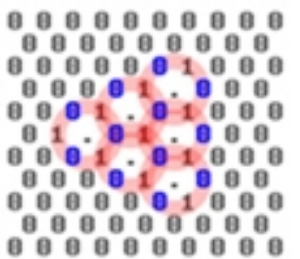

Iteration 3

Supplementary Figure 1 | Closed and segmented population autowaves. a, The closed autowaves were formed due to the hexagonal neighbourhood on the hexagonal lattice. $\mathbf{b}$, The gap segmented autowaves were formed due to the tripod neighbourhood on the hexagonal lattice. These gapes arise owing to smaller number of offsprings per individual (moderate fecundity). Gaps of segmented autowaves are marked by blue colour.

' 0 ' - a free microhabitat that can be occupied;

' 1 ' - a microhabitat with a living individual of a species;

' - a regeneration state of a microhabitat.

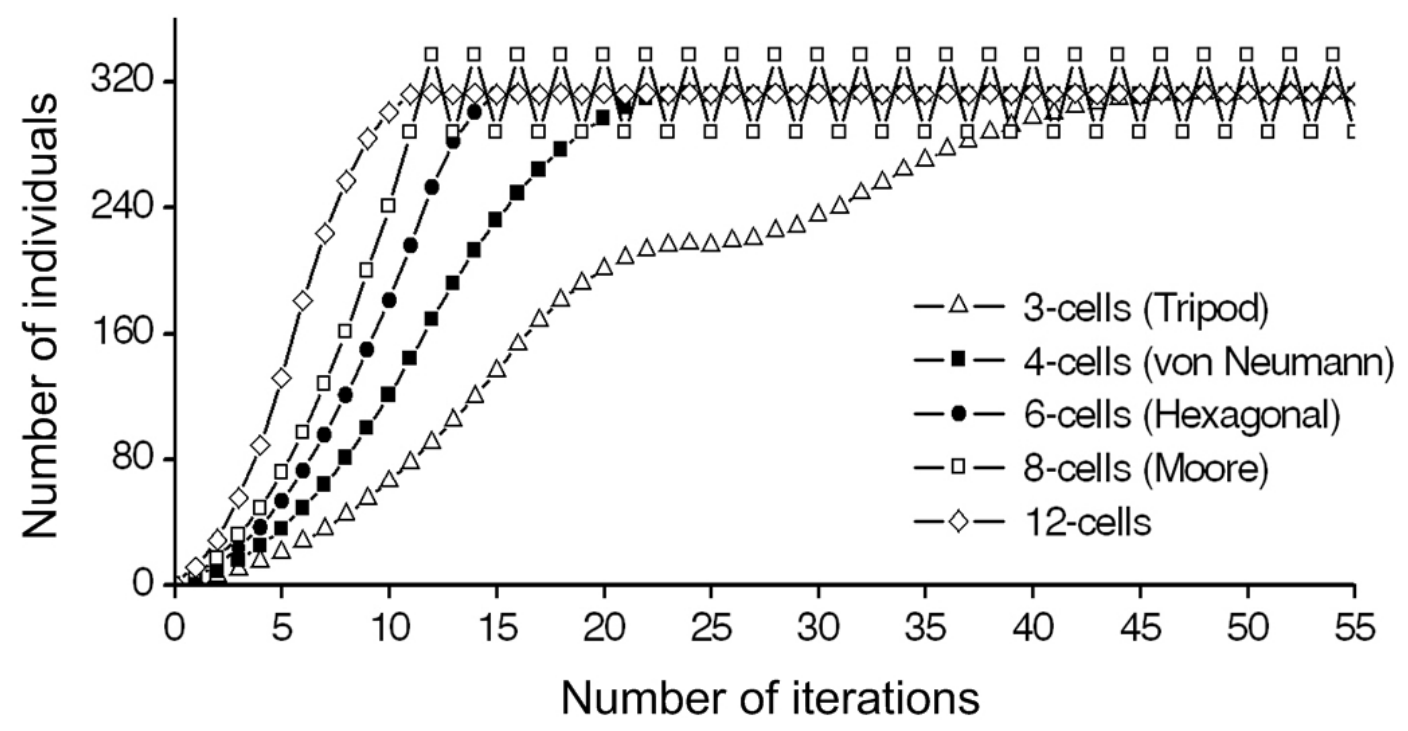


Supplementary Figure 2 | Colonisation of a free ecosystem by one asexually propagating species - five cases when species differ in fecundity rates. The fecundity rate gradation is set by type of neighbourhood: low (3 cells), moderately low (4 cells), normal (6 cells), moderately high ( 8 cells), and high (12 cells). Lattice is hexagonal of $25 \times 25$ cells. One initiating individual is placed on the lattice at the beginning of colonisation in all cases. All neighbourhoods are used on the hexagonal lattice. 


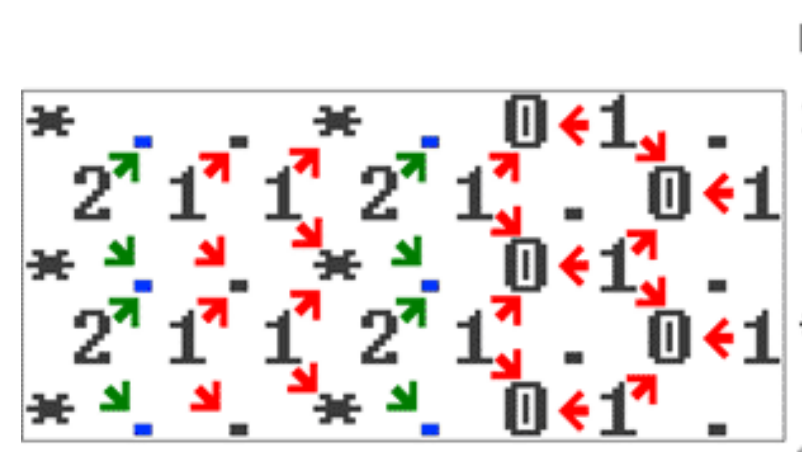

- Free microhabitat that can be occupied by offsprings of any species.

1 - A living individual of the dominant species.

2 - A living individual of the recessive species.

- A regeneration state of a microhabitat after living of an individual of the dominant species.

3. - A regeneration state of a microhabitat after living of an individual of the recessive species.

- The standard directions (red arrows) for possible propagation of individuals of the both neighbourhood).

\section{Supplementary Figure 3 | A dynamic pattern of the tunnel penetration of the} recessive individuals through the gaps in fronts of population autowaves of the dominant species. Sites of penetration of individuals of the recessive species on the next iteration are marked blue. It is 31 iteration of the model based on tripod neighbourhood and on the field of $25 \times 25$ cells with standard start conditions. This pattern drives competition towards the balanced coexistence (Supplementary Movie 3). Autowaves of individuals of the recessive species moving from the left to the right, competitively penetrate into the territory formerly colonized by the dominant species. Arrows indicate the direction of propagating of descendants at the next iteration. Green arrows mark the directions of propagation of the recessive individuals, the red arrows of the dominant ones. 


\section{SUPPLEMENTARY DISCUSSION}

One-dimensional cellular-automata models of competition demonstrate the roles of fitness (dominance or recessiveness) and of duration of regeneration states of microhabitats
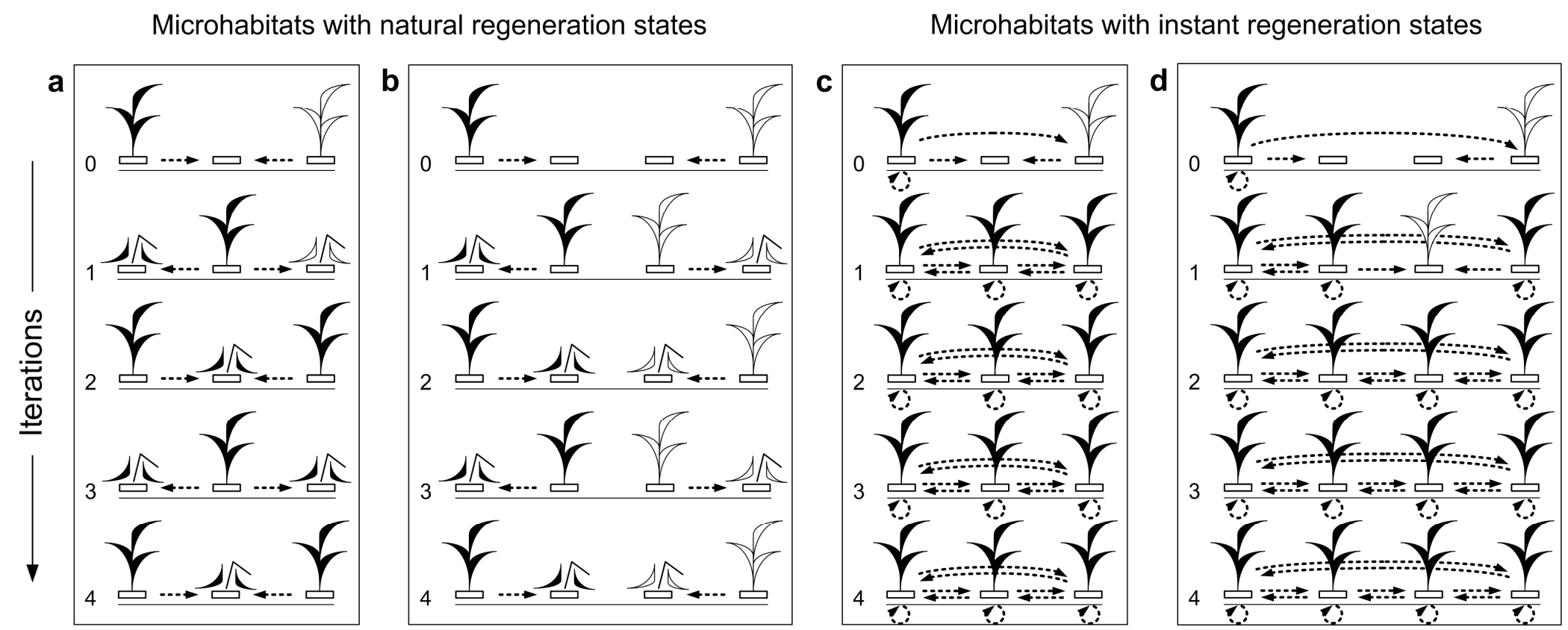

a propagation

direction at

the next iteration

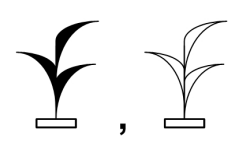

living individuals

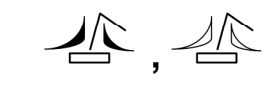

regeneration states

a free microhabitat

Supplementary Figure 4 | One-dimensional models of competition demonstrate the basic cellular-automata rules. The lattices consist of the three $(\mathbf{a}, \mathbf{c})$ and of the four cells $(\mathbf{b}, \mathbf{d})$. Black individuals are dominant and white individuals are recessive. 
The simplest cellular-automata models are shown in pictorial form in Supplementary Fig. 4. Supplementary Fig. 4a,b demonstrate realisation of the basic rules which we also use in two-dimensional models. On these simple onedimensional models we show that the natural regeneration states of microhabitats may enable the coexistence of competitors in one uniform habitat (Supplementary Fig. 4b). This model demonstrates the violation of the principle of competitive exclusion in the small habitat area. Similar models on a more limited area (Supplementary Fig. 4a) or with instant regeneration states (Supplementary Fig. 4c,d) demonstrate competitive exclusion. We mean that 'natural' regeneration is regeneration with duration which is equal to duration of the individual life cycle. We mean that 'instant' regeneration of resources of an ecosystem is artificially instant regeneration by fertilizing, watering, disposal of dead organic matter, etc. The regeneration of microhabitats is the necessary condition for the coexistence of competitors. It follows from the data shown in Supplementary Fig. 4. Additional aspects are a size of the lattice, advantageous positioning of initial competing individuals and the following offspring propagations, fecundity rates (defined by the neighbourhood) and ontogenetic differences. In the models with the instant regeneration states biodiversity disappears (Supplementary Fig. 4c,d). These results speak in favour of maintenance of biodiversity through the natural regeneration states of microhabitats - e.g. deadwood should not be completely removed from forests ${ }^{30}$. However both species may disappear if the time of microhabitats' regeneration is very large. (Supplementary Table 2a).

The dominant species would always eliminate the recessive species in the case if the dominant species has the instant regeneration of microhabitat, but this is unreal and thus this is another argument against the principle of competitive exclusion (Supplementary Figure 4c,d). In the absence of instant regeneration of a microhabitat the coexistence depends on the other autowaves' properties of competing species. 


\section{Advantages of the cellular automata modelling}

A particular advantage of the individual-based cellular automata models is that they are able to reflect holistic spatial-temporal mechanisms of dynamic interrelations among all levels of population ecosystem - between individuals (microhabitats) and their minihabitats, and between their minihabitats and the whole ecosystem (macrohabitat). The advantages of deterministic cellular automata models are in clarity of their cause-effect relationships, the absence of rounding errors (accuracy), in the simplicity of setting boundary conditions, solvability, and holistic relationship of local and global characteristics of models, a detailed mapping of spatial-temporal events. An important feature of this modelling approach is the need for formulation of adequate axioms, based on properties of modelling objects. Correct formulation of the basic interpretations requires a deep and holistic objective knowledge of simulated phenomena. The lattice size, initial cell-states pattern of the whole cellular-automaton field (initial pattern), the type of a neighbourhood and the rules of transitions between states of elementary cell are the key parameters in the cellular-automata modelling. In this paper, we pay much attention to the development of basic axioms of the cellular automata models for providing their conformity to the modelling objects. The main interpretations of the basic axioms are described in Glossary of terms of Supplementary Methods. We have divided competitive contribution of direct domination, fecundity rate and strategic positioning from consuming features of competing species. The presented method can be used in different areas of modelling competition and coexistence of agents of any nature. The deterministic cellular automata modelling may be considered as a simple universal inference automaton of mechanistic insight into studied phenomena as it is based on a detailed visualisation of dynamics of all interrelated processes of a complex system under study. 


\section{Applicability of the cellular-automata for modelling a lawn grasses competition}

The competitive colonisation of small territory by two phenotypically similar species of lawn grasses Poa pratensis L. and Festuca rubra L. ssp. Rubra was an initial biological prototype for us. It was assumed that the lawn is mowed to sustain vegetative propagation. Poa pratensis L. and Festuca rubra L. ssp. rubra are among the most persistent cool-season grasses. It is known that Poa pratensis L. is more resistant to trampling, frost and heat, and Festuca rubra $\mathrm{L}$. ssp. rubra is more resistant to salinity, soil acidity, drought, shade and fungal diseases(28). They are often planted together because they have large similarities in appearance, but differ on stability to various factors of environment. This approach provides a high adaptive flexibility of the lawn. Both grasses actively propagate by rhizomes (Glossary of terms in Supplementary Methods). Two seedling plugs (one tiller per one plug) of the two competing species were placed on a free field of the cellular automaton (one plug per one cell). According to our field observations the cellular automata field simulates an area of a real grassplot of approximately $625 \mathrm{~cm}^{2}\left(1 \mathrm{~cm}^{2}\right.$ per tiller $)$. At the beginning of colonization potentially competing grass species propagate without an interspecies competition. When they meet, the competing territory will be captured by individual of the dominant species. The number of recessive individuals begins to fall until the complete extinction (Fig. 1a and Supplementary Movie 1). The results are similar to the experimental data of Gause(2) relating to changes in the number of individuals in the mixed populations of Paramecium caudatum and Paramecium aurelia on the medium with the lowest concentration of food resources. On the next step, we modelled a single change of the environmental conditions before extinction of the recessive species when only one individual of the recessive species remained on the field (Fig. 1b). An acid rain, infection(29), weather variability, and others environmental factors may change parameters of the environment. The change of environmental parameter may leads to a changeover of dominance if a recessive species is more adapted to new conditions. The formerly recessive species becomes dominant and the number dynamics of individuals changes to the opposite direction. Species which became recessive vanishes from the field if new conditions last for a long time. The formerly dominant species dies after nineteen iterations after the moment of changing external conditions (Fig. 1b). An unusually hot summer, an unusually cold winter, invasion of plant pests are of relatively small periods in the life of perennial grasses. New circumstances appear again and again. Therefore the status of a dominance of competing species may change again and again. In Fig. 1c changes of environmental conditions change the dominance status of species after every four iterations. The result of modelling experiment with changeover of dominance at each iteration is 
shown in Fig. 1d. The relative fluctuations of species numbers are minimal in the last case. That is, the more frequently environmental conditions change, the more stable the diversity of species is. This mechanism ensures stability of a lawn. We have shown that result of competition depend on environmental changes on the cases of two aggressively competing grasses. The cellularautomata models allowed to explain unity and complementarities of opposite views about the reasons of a biodiversity of vegetative communities. The mechanism of coexistence of trophically identical but fitness different species was indicated by Hutchinson in 1941(23). He suggested that the reason of phytoplankton diversity is changing of relevant factors of the environment (e.g. of weather) what blocks the implementation of the principle of competitive exclusion. In further, Hutchinson's point of view was also supported by other researchers $(21,30,31)$. Changeover of dominance owing to environmental changes is one of the reasons of the biodiversity maintaining. Among external environmental factors can be seasonal cycles, weather, illness, predators, changes in the level of food resources. Changeover of dominance can be one of mechanisms of the ecological identity, postulated by Hubbell(7). Really, species coexisting owing to frequent changes of domination have similar and, probably, identical probabilities of existence on a sufficiently long period of time. We conclude here that the cellular automata models reflect a holistic spatialtemporal dynamics of organisms, their ecosystems, and their global ecosystem in simple and evident form.

\section{Applicability of the cellular-automata for modelling a duopoly market in economics}

We suppose that our models are of a wide interdisciplinary interest. Let us consider the interpretation of the models for an economic duopoly market - for a competition between two firms, making similar goods and alternately dominating in the market. The examples of such duopoly are: Intel vs. AMD (the central processors), Coca-Cola vs. Pepsi (the Cola beverages) and others. Let's explain the bio-economic analogy in more details. Let every cell of the cellular automaton model of economic duopoly be a one buyer (one potential sale) on the market and the cellular automaton field be the market as the whole. Occupation of a cell means the acquisition by buyer of a product of one of two competing firms. The bought product is passing a cycle of using from sale until the end of the warranty period. During this cycle the cell is in the active states ' 1 ' or ' 3 ' depending on the firm, and passes at the next iteration into the passive states (states after a warranty period) ' 2 ' or ' 4 ' (according to the firm). After the warranty period during the next iteration at the cell states ' 2 ' and ' 4 ', a product passes the last part of its life cycle including its utilization as waste disposal. Cells in states ' 2 ' and ' 4 ' pass at the next iteration into the free state ' 0 ' or again in ' 1 ' or ' 3 ' state 
(Supplementary Fig. 6a). It is possible to consider this state as the refractory state of preparation of the buyer to new purchase. After the end of product's life cycle, the product owner needs to purchase a new or the same product of this type again. The whole product market we identify as the whole cellular automaton field. Competition between firms is manifested in the fact that when there is an alternative choice for buyers for purchase a product of competing firms, they choose a product of a dominant firm. Wavelike spread of purchases reflects the consistently growing sales in the market. We consider production and management as an internal environment of a firm. The market relates to the external environment of competing firms. Evolution of production technologies and firm management is analogous to evolution of a biological genome. Technological improvements of competing firms in the production and the management may lead to changeover of dominance between the firms. Changes of the market conditions may be another cause of such changeover of dominance between them. It may be proposed on the basis of results presented in Fig. 1a-d, that aggressively competing firms in the absence of cooperation are able to coexist only owing to fairly frequent changes of conditions of their environment, including improvement technologies of production and management and also market changes.

Without changeovers of dominance and in absence of cooperation, competing firms also may co-exist (Fig. 2a, Supplementary Movie 3). The coexistence is possible if the dominant firm not completely satisfy market demand. 
Supplementary Table 1 | Surviving of two competing species as a result of variations of two parameters: (i) dominance (fitness) and (ii) fecundity (neighbourhood)

\begin{tabular}{|c|c|c|c|c|c|c|c|c|c|}
\hline \multicolumn{2}{|c|}{$\begin{array}{l}\text { Rules of transitions } \\
\text { between the states of } \\
\text { a microhabitat* }\end{array}$} & \multicolumn{2}{|c|}{$\begin{array}{c}\text { Moore } \\
\text { neighbourhood }\end{array}$} & \multicolumn{2}{|c|}{$\begin{array}{l}\text { von Neumann } \\
\text { neighbourhood }\end{array}$} & \multicolumn{2}{|c|}{$\begin{array}{c}\text { Hexagonal } \\
\text { neighbourhood }\end{array}$} & \multicolumn{2}{|c|}{$\begin{array}{c}\text { Tripod } \\
\text { neighbourhood }\end{array}$} \\
\hline \multirow{3}{*}{ Species1 } & \multirow{3}{*}{ Species2 } & \multicolumn{8}{|c|}{ Dominant species } \\
\hline & & 1 & 2 & 1 & 2 & 1 & 2 & 1 & 2 \\
\hline & & \multicolumn{8}{|c|}{ Survivor species } \\
\hline $\begin{array}{l}1 \rightarrow 2 \\
\rightarrow 1\end{array}$ & $\begin{array}{l}3 \rightarrow 4 \\
\rightarrow 3\end{array}$ & 1 & 2 & 1 & 2 & 1 & 2 & Both & Both \\
\hline
\end{tabular}

\section{The states of a microhabitat:}

' 1 ' - a living individual of the first species;

' 2 ' - a regeneration state of a microhabitat after living of an individual of the first species;

' 3 ' - a living individual of the second species;

' 4 ' - a regeneration state of a microhabitat after living of an individual of the second.

Two individuals of both species were placed on the field of $25 \times 25$ cells at the initial iteration (at various placements of first two individuals the same results were obtained). At the initial iteration of cellular automaton, two individuals of both species were placed on the field of $25 \times 25$ cells. Evolution of the cellular automata was investigated and results of competition are shown in the table. Transitions between an individual's states are shown by the solid arrows. States in which an individual propagates are shown by the dotted arrows. The graph with all possible transitions is shown in Figure 6a. The dominant species wins at all investigated neighbourhoods except the tripod, where coexistence is observed. This case is the strong violation of the Gause's principle of competitive exclusion. All neighbourhoods are used on the hexagonal lattice. 
Supplementary Table 2 | Surviving of two competing species as a result of variations of three parameters: (i) type of ontogenetic development, (ii) dominance (fitness) and (iii) fecundity (neighbourhood)

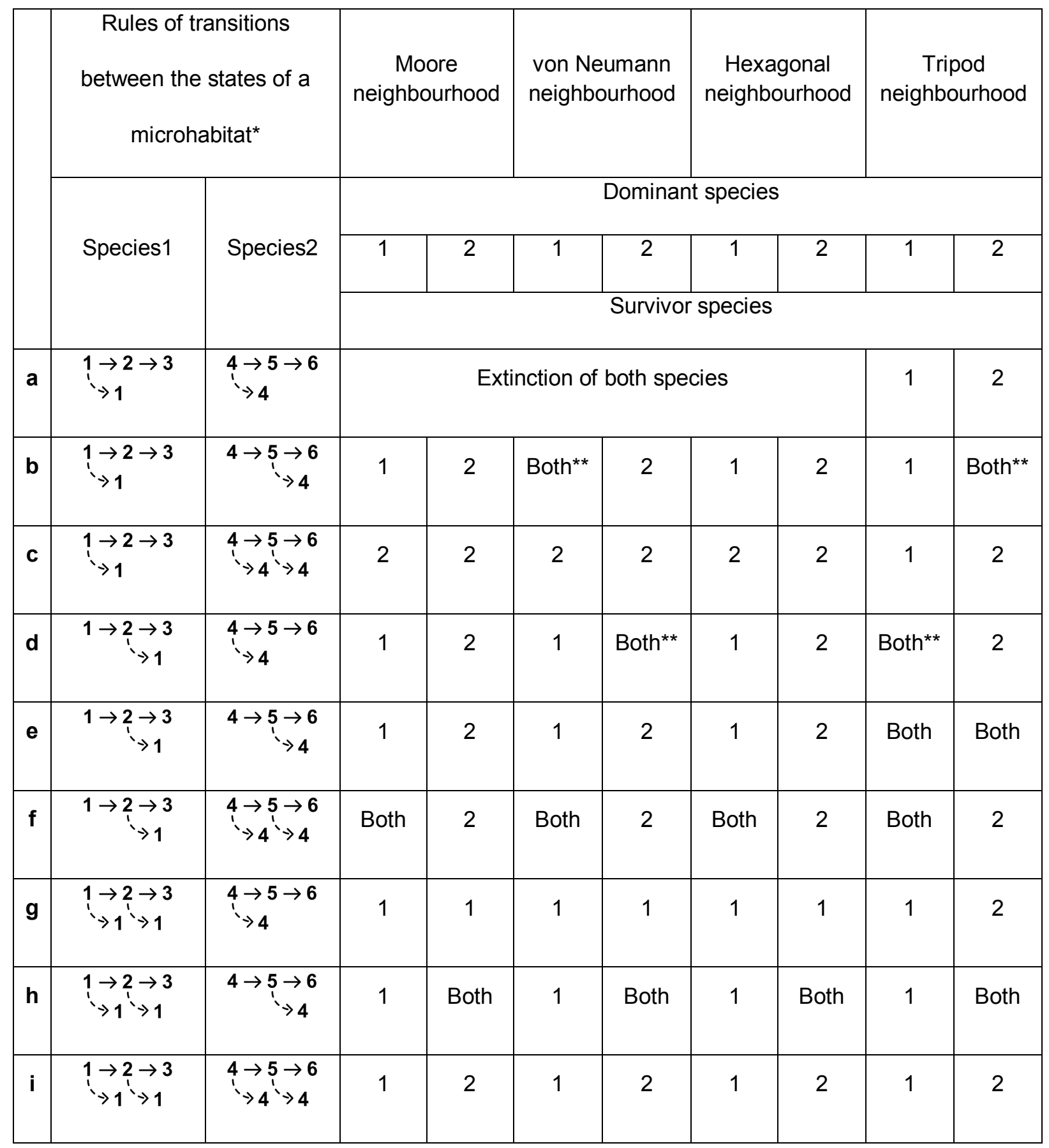

* - by the solid arrow is shown in what state an individual will pass on the following iteration and by the dotted arrow is shown in what state an individual propagates. ** - the result of competition depends on the initial placement of individuals on the field. 
States of a microhabitat:

' 1 ' - a living individual of the first species.

' 2 ' - a living individual of the first species.

' 3 ' - a regeneration state of a microhabitat after living of an individual of the first species.

' 4 ' - a living individual of the second species.

' 5 ' - a living individual of the second species.

' 6 ' - a regeneration state of a microhabitat after living of an individual of the second species.

Two individuals of both species were placed on the field of $25 \times 25$ cells at the initial iteration (at various placements of first two individuals the same results were obtained except the cases marked with two asterisks). In the case of a tripod neighbourhood with propagation of both species in the second ontogenetic stages their coexistence is observed - Supplementary Table 2e. This case is also the violation of the principle of competitive exclusion. The transition graph between all states is presented in Supplementary Fig. 6b. All neighbourhoods are used on the hexagonal lattice.

\section{SUPPLEMENTARY METHODS}

The cellular-automata modelling of ecosystem. Deterministic cellular automata were used for modelling the spatio-temporal dynamics of simultaneous colonisation of a small ecosystem by two trophically identical but fitness different competing species. An integral ecosystem (macrohabitat) is modelled by a whole cellular automaton. The lattice is closed to a torus by periodic conditions for avoiding boundary effects. The hexagonal lattice was used, because it most naturally corresponds to the principle of densest packing of round projections of microhabitats. Each cell models a microhabitat which in a free state contains resources for existence of any one individual. A life of an individual lasts a one iteration of the automaton. All states of microhabitats have the same duration. All microhabitats are identical i.e. every individual of both species consumes identical quantity of identical resources. Our standard cellular automaton consists of regular lattice of $25 \times 25$ cells, each of which may be in one of the 5 states. Initiation of the population is carried out by the placement of two single individuals of competing species. The initiate individuals of the two competing 
species were placed in the coordinates $(10,10)$ for the first and $(14,14)$ for the second species. Colonisation of ecosystem and dynamics of competition occur as successive waves of changes of the cells' states.

Each microhabitat can be in one of the five kinds of states: '0'- a free microhabitat that can be occupied by offsprings of any species; ' 1 ' - a living individual of the first species; '.- a regeneration state of a microhabitat after living of individual of the first species; ' 2 ' - a living individual of the second species; ' '” - a regeneration state of a microhabitat after living of individual of the second species. These symbols are used in Supplementary Movies 1-4. A microhabitat cannot be populated immediately after an individual's death because the recovery of microhabitat's resources must occurs (regeneration state), but it can be occupied immediately after regeneration when it passed into the free state. Rules for transitions between states of the cellular automaton are defined as simple logical 'if-then' statements. Possible transitions between the states of a cell are represented on the directed graph (Supplementary Fig. 6).

Dominance. One of the two species is dominant at each iteration. The dominance is defined as the primary ability of individuals of the species to occupy a free microhabitat in a conflict of interests with an individual of another species (Supplementary Fig. 4a). The sequence of checking 'if-then' statements of the birth rules realisation defines the dominance. Changeover of dominance is modelled using dynamically switching rules of the cellular automaton.

Each individual can be represented as a pacemaker, which generates a population autowave on the next iteration of the cellular automaton. In this case, the propagation of offsprings occurs into cells determined by the neighbourhood. The size of the neighbourhood defines the species' fecundity. Various types of neighbourhoods are represented on Supplementary Fig. 7.

A two-stage ontogeny of individuals is modelled by introducing one additional state for each species, prolonging the lifetime of individuals of both species up to two iterations, on each of which their propagation can be possible or not. The number of microhabitats' states is equal 7 when we model an additional phase of ontogeny (Supplementary Fig. 6b).

Autowave interpretations. Autowave nature of population dynamics based on the fact that every individual is the source of autowave (Supplementary Fig. 1). The cellular automata models allow to trace a fate of every individual, their local interactions and the result of competition at the ecosystem level as a whole. Reproduction of individuals of competing species occurs in the form of 
propagating waves of their synchronous reproduction, life, death and subsequent regeneration of microhabitats (Supplementary Fig. 1). These population waves are autowaves because they are self-sustaining waves of changes in states of the active medium, which are accompanied by energy dissipation. The energy is consumed for life support of individuals and for regeneration of an ecosystem. The autowave interpretations allow us to give a general characterisation of the investigated processes, because autowaves' properties are universal and independent of specificity of systems where they are implemented.

\section{Glossary of Terms}

The glossary of terms was used in design and interpretations of our model.

The terms: Active medium, Autowaves, Biological diversity, Cellular automaton, Changeover of dominance, Dominance, Duopoly, Dynamically switching rules, Ecosystem, Fecundity, Fecundity rate, Fertility, Fertility rate, Field of the cellular automaton, Fitness, Habitat, Individual, A limiting factor of the environment, Macrohabitat, Mechanistic mechanisms, Microhabitat, Minihabitat, Pacemaker, Phenomenological models and phenomenological mechanisms, Recessiveness, Rhizomes, Tiller, Uniform cellular automaton, White box model.

Active medium - a medium that contains distributed resources for maintenance of autowaves and capable to regenerate its properties after passage of autowave in a result of pumping energy from outside. Ecosystem (habitat) is an example of an active medium, and life activity of species individuals is an example of an excited state of the environment.

Autowaves - self-sustaining waves of changes in states of the active medium, which are accompanied by energy dissipation. Autowaves properties are universal and independent of specific systems where they are implementing.

Biological diversity or biodiversity in the narrow sense (used here) means the number of different species living within the particular biotope.

Cellular automaton. Ecologically speaking, the cellular automaton is a spatiotemporal model of interrelations between each of all individual organisms and of the whole ecosystem. Integration of cells (microhabitats) into the united whole ecosystem (macrohabitat) is carried out through defined neighbouring of each cell (minihabitat). More formally speaking, cellular automaton is a polyautomaton, which is a quadruple of objects $<\mathbf{G}, \mathbf{Z}, \mathbf{N}, \mathbf{f}>$, where

$\mathbf{G}$ is a lattice of a cellular automaton consisting of an array of elements;

$\mathbf{Z}$ is a finite set of possible states of the elements of the lattice;

$\mathbf{N}$ is a cellular automata neighbourhood;

$\mathbf{f}$ is a function of transitions between states of a cell. 
A one iteration of the cellular automaton consists of applying the transition function to each element of the lattice. The iteration is completed only after the new state will be defined for all of its elements. A lattice of a cellular automaton consists of cells, which are identical automata and each of which may be in a predetermined number of discrete states. States of each of all cells are iteratively updating synchronously at discrete time steps according to local rules. A neighbourhood relation is defined over the lattice, indicating for each cell which cells are considered to be its neighbours. A neighbourhood consists of a cell and its defined neighbours. All cells have neighbourhoods of the same shape and the same rules for updating. These transition rules logically connect a state of each cell and states of its neighbouring cells on the given iteration with the state of the cell on the next iteration. In general cellular automaton can be considered as a function that iteratively transforms an initial cell-state pattern of the whole cellular-automaton (a start states pattern) field into subsequent configurations. Neighbourhoods that are used in the models of the given work are presented in Supplementary Fig. 7.

Changeover of dominance is ecological situation when owing to change of external or internal environment, a former recessive species became the dominant and a former dominant species became the recessive. Changeover of dominance is modelled through programming of dynamically switching rules. It is one of mechanisms of maintaining biodiversity in the evolutionary struggle for existence.

Dominance is defined as the primary ability of an individual to occupy a free microhabitat in a conflict of interests with an individual of another species. Antonym: recessiveness.

Duopoly is a specific type of a market form where a market or an industry is dominated by two sellers. It is competition between two firms, which make similar goods and alternately dominate in the market. Examples of such duopoly are: Intel vs. AMD (the central processors), Coca-Cola vs. Pepsi (the Cola beverages) and others.

Dynamically switching rules are the cellular automaton rules based on dynamically switching functions. The method of dynamic switching rules allowed to simulate the environmental changes that lead to changeover of species dominance. We suppose that the given approach is applicable to any cellular automata and allows to implement different rules for different iterations.

Ecosystem is the unity of all interacting organisms and of all their habitats within a specific area.

Fecundity is the potential for reproduction - the potential ability to produce offsprings of an individual or population.

Fecundity rate is a potential per capita offsprings' number on a next iteration. 
Fertility is the actual production of offsprings - the actual reproductive rate of an individual or population.

Fertility rate is an actual per capita offsprings' number on a next iteration.

Field of the cellular automaton is the ordered set of cells ("microhabitats", finite-state machines) where each cell corresponds to certain site of a uniform, regular lattice.

Fitness is ability of individual to maintain its health, well being and directly competes for resources of environment. It is expressed in domination and describes how successful an organism is at leaving offspring compared to its competitors.

Habitat is the intrinsic environment (the specific natural home) where a part of a species is able to survive. Habitat is a more general and less concretised term than 'microhabitat', 'minihabitat' and 'macrohabitat' are (see the corresponding definitions of the Glossary and Supplementary Fig. 5, concreting the notion 'habitat' in the cellular automata context).

a

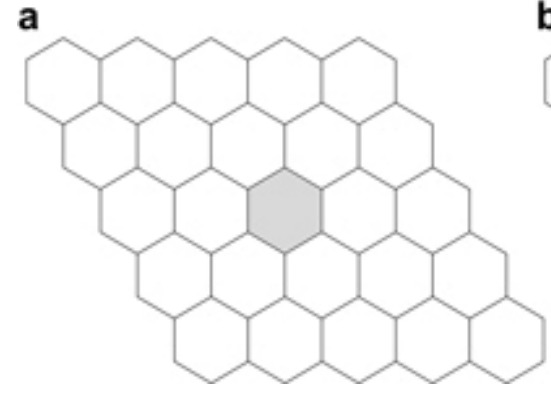

b

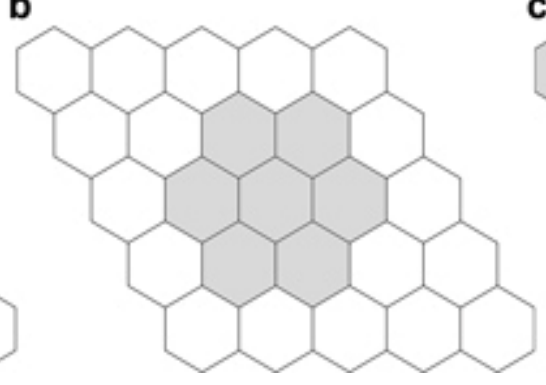

C

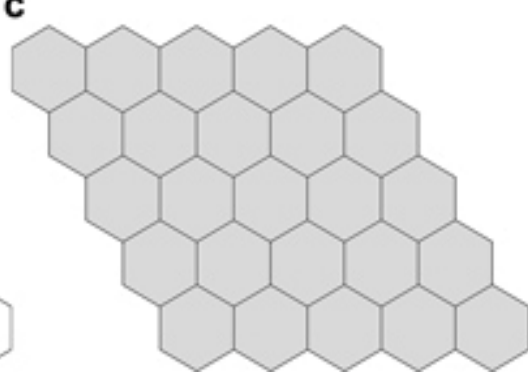

Supplementary Figure 5 | 'Microhabitat', 'minihabitat' and 'macrohabitat' on example of hexagonal neighbourhood on hexagonal lattice. The grey colour indicates: a, A microhabitat (any one cell of the field). b, A minihabitat (microhabitat and all the adjacent neighbouring cells). c, A macrohabitat (all field of the cellular automaton). The field consists of $5 \times 5$ cells and is used here as example.

Individual is one organism of a species (an autonomous agent in the most general case).

A limiting factor of the environment is the environmental factor that controls an ability of the species individuals to thrive and to reproduce itself. These ecological factors limit bioefficiency (bioproductivity) of organisms of the species when these factors are scarce.

Macrohabitat is the total environment where individuals may inhabit and propagate actually and potentially. Here, in the cellular automaton model, 
macrohabitat is the entire field of the cellular automata. Macrohabitat contains a complete set of all cells assigned to the sires of the cellular automaton lattice (Supplementary Fig. 5c).

Mechanistic mechanisms permit most clearly to understand a system under study and to predict its behaviour. We consider mechanistic mechanisms as consisting of two constituents - (1) causes-effects relations and (2) part-whole relations. The causes should be sufficient to understand their effects and the parts should be sufficient to understand the whole. In our research the "whole" is an ecosystem with populations of competing species. The "parts" are: individuals, microhabitats and interactions between the individuals and their immediate environment (neighbourhoods). We believe that the "causes" being investigated here are extreme principles of physics and in particular the Lotka's principle of maximum energy flux and the second law of thermodynamics. The "effects" are results of the interspecific competition. A deterministic, individual-based logical cellular-automata method developed in this study is the most suitable tool for search of the mechanistic mechanisms of complex systems. It is the white-box modelling method based on physically interpreted ecological axioms. These cellular automaton works as a visualising inference automaton of mechanistic insight into studied phenomena. It provides reducing of complex holistic dynamics of interspecific competition to the level of simple logical axioms. Microhabitat is the intrinsic physical environment where a particular individual inhabits. A microhabitat is a totality of all environmental conditions which are necessary for individual's life (e.g. of a grass unit) and place where regeneration of the resources is possible. Here, in the cellular automaton model, a microhabitat is each discrete cell of the cellular automata field which corresponds to a specific site of the lattice of the cellular automaton. A cell represents a place which can be occupied by an individual autonomous agent (e.g. by an individual of a species) and contains the necessary resources for its individual life (Supplementary Fig. 5a).

Minihabitat is the intrinsic physical environment (the specific natural home) where a particular individual of a species is able to propagate in one generation. Here, in the cellular automaton models a minihabitat is a cell together with its neighbouring cells. (Supplementary Fig. 5b).

Pacemaker - leading centre, auto-oscillation area in excitable (active) medium a source of local self-oscillations which generates diverging autowave. Within the limits of autowave interpretations each separate organism, capable to autonomous reproduction may be considered as a pacemaker of population autowave.

Phenomenological models and phenomenological mechanisms showing what happens with modelled object on a macro-level but does not show how it happens on a mini- and a micro-level. They describe some empirical observations, but have no foundations in mechanisms or first principles, making difficult a 
prediction, generating new knowledge and the creation of new technologies. Phenomenological models are "black-box" models what means that they are not transparent for understanding of mechanisms of simulated objects.

Metaphorically speaking, the phenomenological model is analogous to a picture of the studied phenomenon, drawn with the help of mathematical apparatus.

Earlier Tilman noted that models of population dynamics based on differential equations are phenomenological and therefore hinder mechanistic understanding of phenomena under study (32).

Recessiveness is defined as the secondary (inferior) ability of an individual to occupy a free microhabitat in a conflict of interests with an individual of another species. Antonym: dominance.

Rhizomes are horizontal creeping underground shoots by means of which certain plants vegetatively (asexually) propagate themselves. Unlike a root, rhizomes have buds, nodes, and scaly leaves. Rhizome develops tillers with roots and leaves at nodes along its length.

Tiller is a minimal relatively autonomic grass shoot that sprouts from the base of grass and which is able to propagate.

Uniform cellular automaton applies the same rules for each cell.

White box ("clear box", "glass box", "open box") model has a "transparent walls" - all events at all levels of the simulated dynamic system and at all stages of its dynamics are directly visible (Supplementary Figure 7). White box model directly shows mechanistic mechanisms of the studied phenomena. It allows to obtain a new physical understanding of the studied system and to predict its future development. In the literature on ecological modelling the white-box modelling is discussed as a future opportunity(33). Currently, this type of modelling is used and widely discussed only in engineering sciences(34-38). We consider the cellular automata method developed in this study as the white box modelling. 

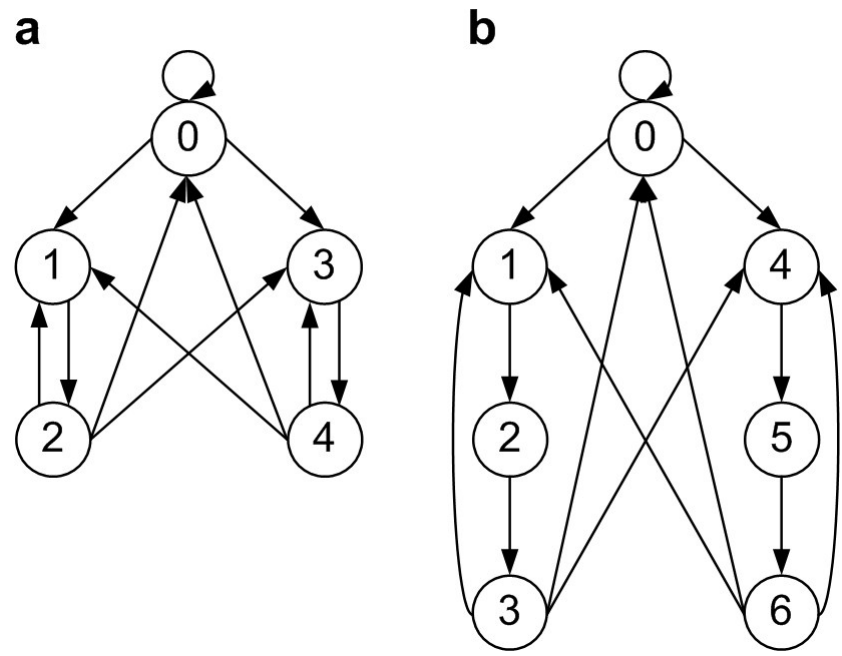

Supplementary Figure 6 | State transition graphs of the cellular automata models.

\section{a, Standard cellular automata:}

' 0 ' - a free microhabitat that can be occupied by an offspring of any species.

' 1 ' - a microhabitat is occupied by a living individual of the first species.

' 2 ' - a regeneration state of a microhabitat after living of an individual of the first species. In Supplementary Movies this state is represented as symbol '.'

' 3 ' - a microhabitat is occupied by a living individual of the second species. In Supplementary Movies this state is represented as symbol ' 2 '.

' 4 ' - a regeneration state of a microhabitat after living of an individual of the second species. In Supplementary Movies this state is represented as symbol '*'.

\section{b, Cellular automata with additional ontogenic stages:}

' 0 ' - a free microhabitat that can be occupied by offsprings of any species.

' 1 ' - a microhabitat is occupied by a living individual of the first species ( 1 st ontogenic stage).

' 2 ' - a microhabitat is occupied by a living individual of the first species $\left(2^{\text {nd }}\right.$ ontogenic stage).

' 3 ' - a regeneration state of a microhabitat after living of an individual of the first species.

' 4 ' - a microhabitat is occupied by a living individual of the second species $\left(1^{\text {st }}\right.$ ontogenic stage).

' 5 ' - a microhabitat is occupied by a living individual of the second species $\left(2^{\text {nd }}\right.$ ontogenic stage).

' 6 ' - a regeneration state of a microhabitat after living of an individual of the second species. 
a

\begin{tabular}{|l|l|l|}
\hline$i-1, j-1$ & $i-1, j$ & $i-1, j+1$ \\
\hline$i, j-1$ & $i, j$ & $i, j+1$ \\
\hline$i+1, j-1$ & $i+1, j$ & $i+1, j+1$ \\
\hline
\end{tabular}

e

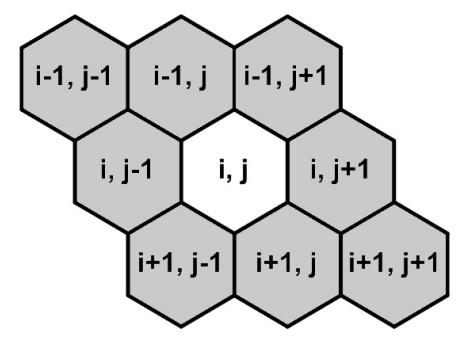

b

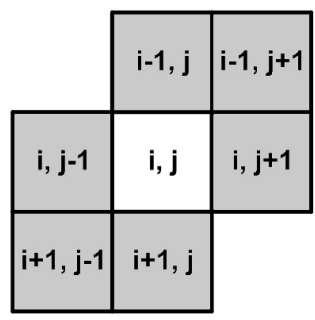

f

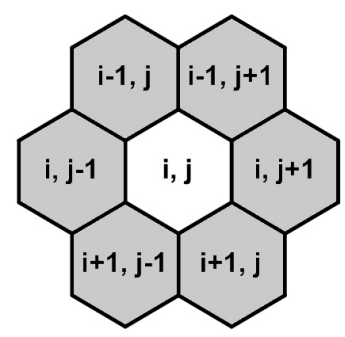

C

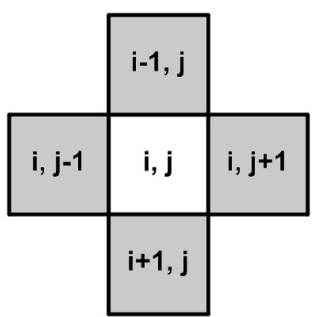

g

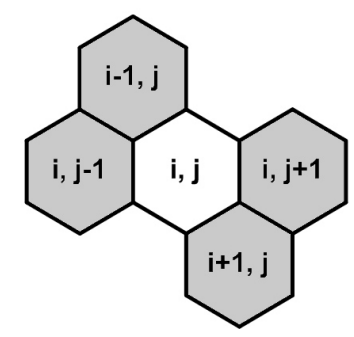

d

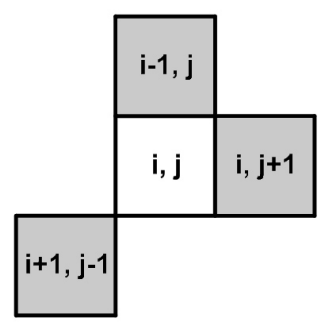

h

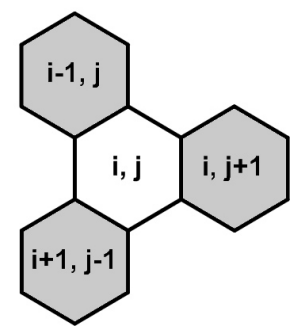

\section{Supplementary Figure 7 | Various types of neighbourhoods of the cellular}

automaton. (a-d), neighbourhoods on the square lattice - Moore, hexagonal, von Neumann and tripod. (e-h), the same neighbourhoods, but on the hexagonal lattice. On each iteration for each site with coordinates $(i, j)$ of a lattice its neighbourhood is studied, taking into account periodic boundary conditions. The neighbourhoods on the hexagonal lattice are used for modelling fecundity rate of individuals.

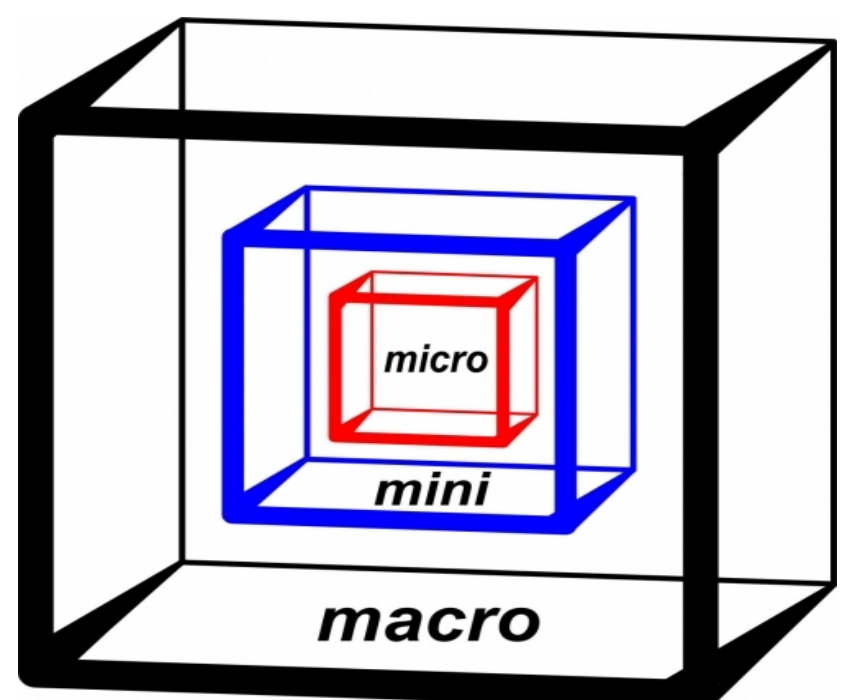

Supplementary Figure 8 | Figurative representation of white-box model. All events are visible on the macro-, mini- and micro-levels. 


\section{Supplementary Table 3 | Parameters of the cellular-automata models which are presented in the Figures 1, 2, and 3}

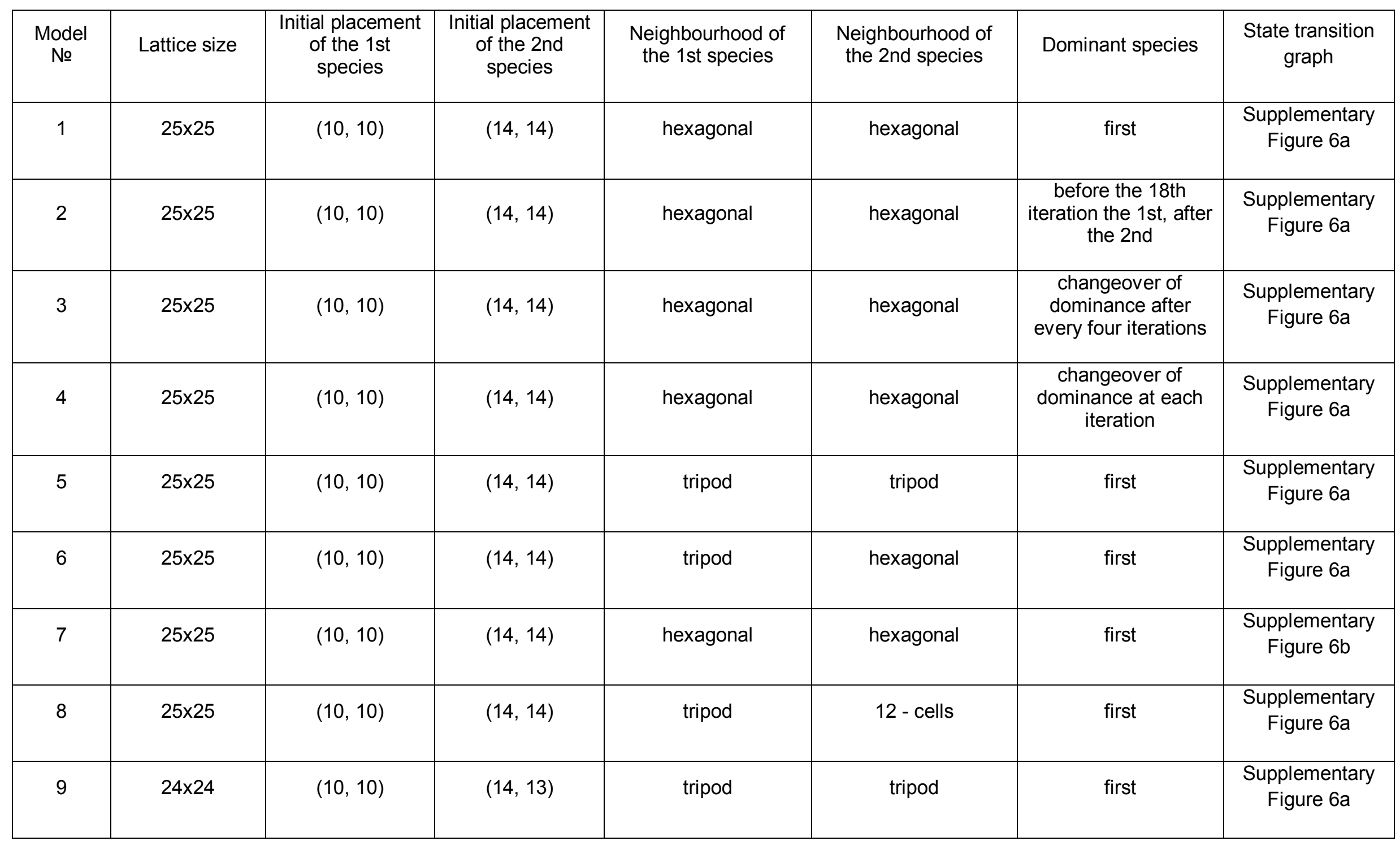




\section{SUPPLEMENTARY MOVIE LEGENDS}

The symbols of the five states of a microhabitat, which are used in the movies: ' 0 ' - a free microhabitat that can be occupied by an offspring of any species;

' 1 ' - a microhabitat is occupied by a living individual of the dominant species 1 ;

' - the regeneration state of a microhabitat after living of an individual of the first species;

' 2 ' - a microhabitat is occupied by a living individual of the recessive species 2 ;

' $*$ ' - the regeneration state of a microhabitat after living of an individual of the second species.

\section{Movie S1}

The first movie shows the interspecific competition of trophically identical but fitness different species in stable environmental conditions. This movie corresponds to Fig. 1a. The both species have the hexagonal neighbourhood i.e. they aggressively try to occupy the all nearest environment for propagation. The species ' 1 ' is dominant. The dynamics takes place on the hexagonal lattice of $\mathrm{N}=25 \mathrm{x} 25$ sites. At the initial iteration single individuals of both species are placed on the lattice. Coordinates of the site for the first individual of the species ' 1 ' are $(10,10)$. Coordinates of the site for the first individual of the species ' 2 ' are $(14,14)$. The dominant species ' 1 ' wins the recessive species ' 2 '. The dominant species ' 1 ' drives out the recessive species ' 2 '. It is the case of the competitive exclusion.

The movie is available on YouTube: http://youtu.be/EoxC-YcrHXA?hd=1

\section{Movie S2}

The second movie shows the interspecific competition of the trophically identical but fitness different species ' 1 ' and '2'under environmental fluctuations. The competing species have a changeover of dominance on each iteration of cellular automata. This movie corresponds to Fig. 1d. The both species have the hexagonal neighbourhood i.e. they aggressively try to occupy the all nearest environment for propagation. The dynamics takes place on the hexagonal lattice of $\mathrm{N}=25 \times 25$ sites. At the initial iteration single individuals of both species are placed on the lattice. Coordinates of the site for the first individual of the species ' 1 ' are $(10,10)$. Coordinates of the site for the first individual of the species ' 2 ' are $(14,14)$. The species coexist due to environmental fluctuations that lead to changeovers of dominance. The species coexist due to environmental fluctuations. This model is the most probable explanatory mechanism of the paradox of the plankton.

The movie is available on YouTube: http://youtu.be/QbXQabLtr2U 


\section{Movie S3}

The third movie shows the interspecific competition of the trophically identical but fitness different species ' 1 ' and ' 2 ' in stable environmental conditions. This movie corresponds to Fig. 2a. The both species have the tripod neighbourhood i.e. they have lowered fecundity and as the result are less aggressive in propagation than competing species modelled in the movies 1 and 2 . The species ' 1 ' is dominant. The dynamics takes place on the hexagonal lattice of $\mathrm{N}=25 \times 25$ sites. At the initial iteration single individuals of both species are placed on the lattice. Coordinates of the site for the first individual of the species ' 1 ' are $(10,10)$. Coordinates of the site for the first individual of the species ' 2 ' are $(14,14)$. The species coexist due to the gaps in population autowaves of the dominant species ' 1 '. Strong violation of the Gause's principle of competitive exclusion occurs owing to the low competitors' fecundity.

The movie is available on YouTube: http://youtu.be/gARkN8XDUEQ

\section{Movie S4}

The fourth movie shows the interspecific competition of the trophically identical but fitness different species ' 1 ' and ' 2 ' in stable environmental conditions. This movie corresponds to Fig. 3b. The both species have the tripod neighbourhood i.e. they have lowered fecundity and as the result are less aggressive in propagation than competing species modelled in the movies 1 and 2 . The species ' 1 ' is dominant. The dynamics takes place on the hexagonal lattice of $\mathrm{N}=24 \times 24$ sites. At the initial iteration single individuals of both species are placed on the lattice. Coordinates of the site for the first individual of the species ' 1 ' are $(10,10)$. Coordinates of the site for the first individual of the species ' 2 ' are $(14,13)$. The recessive species ' 2 ' drives out the dominant species ' 1 ' owing to the advantageous positioning of offsprings. The specific size of a lattice is also important. Owing to advantageous offsprings positioning, individuals of the recessive species propagate into microhabitats just before the iteration in which individuals of the dominant species will try to occupy these microhabitats on the next iteration. This strategy of anticipatory deprivation of resources for competitor's offsprings propagation allows individuals of the recessive species to avoid direct competition and to drive out the dominant species of resources for propagation.

The movie is available on YouTube: $\underline{\text { http://youtu.be/KKe_2jznOT8 }}$ 